\title{
Jet Physics at the LHC and the Tevatron
}

\author{
Jay R. Dittmann ${ }^{1, a}$ for the LHC (ALICE, ATLAS, CMS) and Tevatron (CDF, D0) Collaborations \\ ${ }^{1}$ Baylor University, One Bear Place \#97316, Waco, TX, 76798-7316, USA
}

\begin{abstract}
We present a summary of recent jet measurements from the CERN Large Hadron Collider and the Fermilab Tevatron.
\end{abstract}

\section{Introduction}

Jets are produced copiously in hadron-hadron collisions. Measurements of jet observables serve as crucial tests of quantum chromodynamics (QCD) and they provide precise information on the structure of the proton. Furthermore, a careful understanding of processes involving jets can greatly improve the sensitivity of searches for new phenomena. This report summarizes recent jet measurements in four main areas: inclusive jet production, dijet production, jet ratios and the extraction of $\alpha_{s}$, and jet mass and substructure.

\section{Inclusive Jet Production}

Over a period of many years, the CDF and D0 experiments at the Fermilab Tevatron accumulated data from $p \bar{p}$ collisions at $\sqrt{s}=1.96 \mathrm{TeV}$. Recent measurements of the inclusive jet cross section from these experiments $[1,2]$ have confirmed the predictions of perturbative QCD (pQCD) over eight orders of magnitude in $d \sigma^{2} / d p_{T} d y$ for various ranges of jet rapidity $y$. Moreover, these measurements have contributed to the development of many modern parton distribution function (PDF) sets used today at the Large Hadron Collider (LHC).

New inclusive jet measurements at the LHC explore $p p$ collisions at a much higher center of mass energy than the Tevatron. An analysis performed by ATLAS at $\sqrt{s}=$ $7 \mathrm{TeV}$ [3] compares jet cross sections to a next-to-leadingorder (NLO) QCD calculation with non-perturbative (NP) corrections for various jet rapidity ranges out to $|y|=4.4$. In this analysis, data and theory agree over many orders of magnitude, although theory predicts somewhat larger cross sections at large jet $p_{T}$ (see Fig. 1). A new CMS measurement at $\sqrt{s}=8 \mathrm{TeV}$ [4] extends the range of jet $p_{T}$ even higher to $2.5 \mathrm{TeV}$. As shown in Fig. 2, data and theory agree within 5-10\% for various rapidity ranges and PDF sets, except for the ABM11 PDF set for small $|y|$ and $p_{T}>300 \mathrm{GeV}$.

\footnotetext{
ae-mail: Jay_Dittmann@baylor.edu
}

The ALICE collaboration performed a measurement of the inclusive jet cross section at $\sqrt{s}=2.76 \mathrm{TeV}$ [5] for anti- $k_{T}$ jets with two jet size parameters: $R=0.2$ and $R=0.4$. Data agree well with NLO pQCD calculations with hadronization (see Fig. 3). In addition, the ratio of the cross sections for the two size parameters provides a measure of radiation within the jet, which is also well described by theory.

In a recent analysis, ATLAS compared the inclusive jet cross section at $\sqrt{s}=2.76 \mathrm{TeV}$ and $\sqrt{s}=7 \mathrm{TeV}$ [6]. The cross section ratio $\sigma(2.76 \mathrm{TeV}) / \sigma(7 \mathrm{TeV})$ vs. $p_{T}$, which has the benefit of small experimental uncertainties, generally agrees with theory; however, the data are slightly higher than predictions in the central rapidity region and lower in the forward region (see Fig. 4).

\section{Dijet Production}

Recent measurements of the dijet production cross section from $p p$ collisions at $\sqrt{s}=7 \mathrm{TeV}$ by CMS and ATLAS have extended the range of dijet invariant masses to a new high of $5 \mathrm{TeV}[3,7]$. The data are compared to expectations based on NLO QCD calculations with corrections for NP effects. In addition, the ATLAS analysis compares data to parton shower Monte Carlo simulations with NLO matrix elements. In both analyses, data and theory agree to within $10 \%$ for various rapidity ranges and PDF sets (see Figs. 5 and 6). With experimental and theoretical uncertainties that are comparable in size in many regions of phase space, the results are sensitive to different theoretical predictions and can be used to constrain global PDF fits.

ATLAS has measured the flavour composition of dijet events at $\sqrt{s}=7 \mathrm{TeV}$ [8], where the jet flavour is determined by template fits to the kinematic properties of secondary vertices inside jets. Templates differentiate between $b, c$, and light quarks (denoted " $u$ "). The flavour of each of the two jets is determined, yielding six different combinations of flavour pairs. Data and theory agree for most flavour pairs, except for some disagreement in the bottom-light quark fraction at high jet $p_{T}$ (see Fig. 7). 

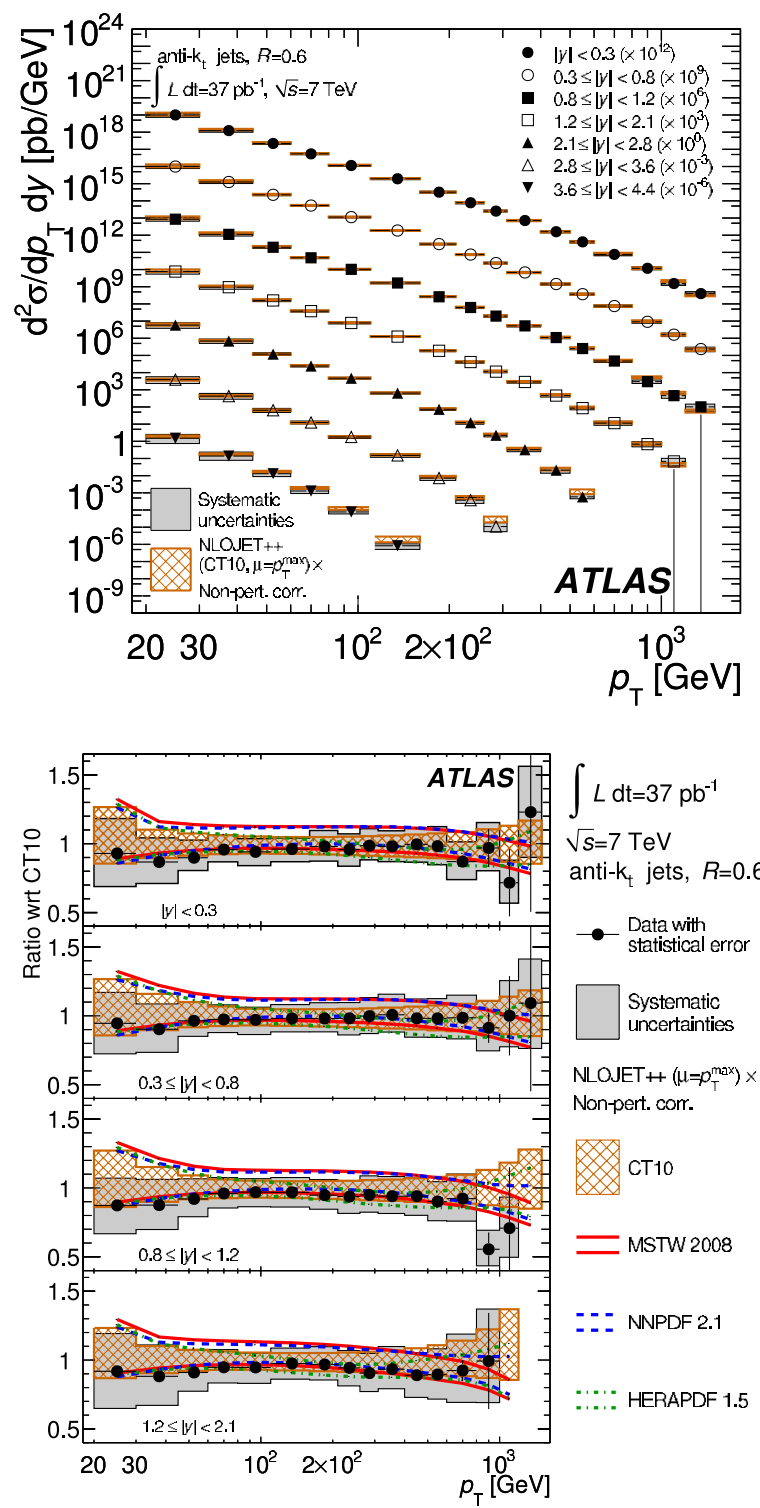

Figure 1. (Top) ATLAS double-differential inclusive jet cross section as a function of jet $p_{T}$ in different regions of $|y|$ for jets identified using the anti- $k_{T}$ algorithm with $R=0.6$, as described in Ref. [3]. (Bottom) Ratios of the inclusive jet cross section to the theoretical prediction obtained using NLOJET++ with the CT10 PDF set. Many additional comparison plots are available in [3].

\section{Jet Ratios and the Extraction of $\alpha_{s}$}

The D0 collaboration has published a "trilogy" of jet ratio measurements from $p \bar{p}$ collisions at $\sqrt{s}=1.96 \mathrm{TeV}$ that probe QCD predictions in different approximations (see Fig. 8). The first, $R_{\Delta R}$ [9], measures the angular correlations of jets. The data for jet $p_{T}>50 \mathrm{GeV}$ are well described by pQCD at NLO in $\alpha_{s}$ with NP corrections. Results for $\alpha_{s}\left(p_{T}\right)$ are extracted and are in good agreement with renormalization group equations for the running of $\alpha_{s}$ for momentum transfers up to $400 \mathrm{GeV}$ (see D0 points in Fig 9). The extracted value $\alpha_{s}\left(M_{Z}\right)=0.1191_{-0.0071}^{+0.0048}$ is in agreement with the world average. The second measurement, $R_{3 / 2}$ [10], is a ratio of the three-jet to two-jet
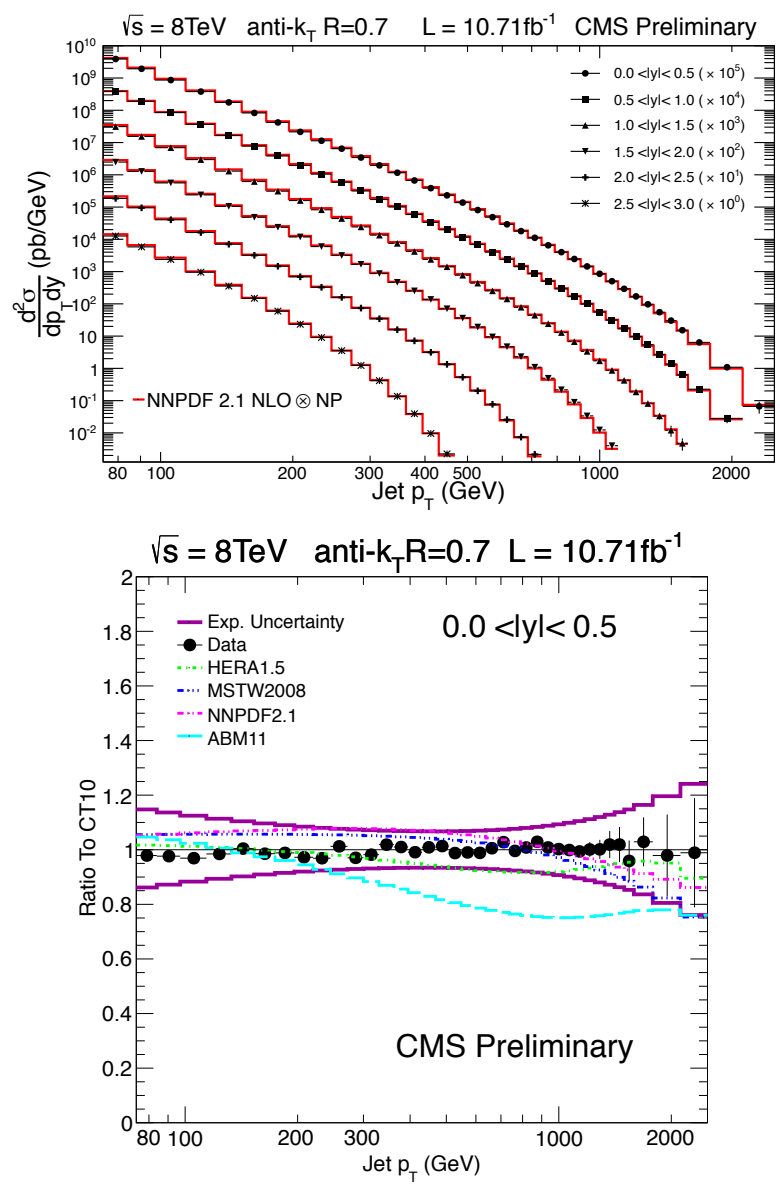

Figure 2. (Top) CMS double-differential inclusive jet cross section compared to NLO predictions with a NP correction using the NNPDF2.1 PDF set, as described in Ref. [4]. (Bottom) Ratio of data over theory at NLO with a NP correction for the CT10 PDF set, together with predictions for four other PDF sets, for the jet rapidity range $0.0<|y|<0.5$. Many additional comparison plots are available in [4].

cross sections as a function of $p_{T \max }$, the highest jet $p_{T}$, for various $p_{T \text { min }}$ requirements. The data are well described by pQCD at NLO in $\alpha_{s}$ with NP corrections. The third measurement, $R_{\Delta \phi}$ [11], measures the fraction of inclusive dijet events in which the azimuthal separation of the two highest- $p_{T}$ jets is less than a specified value of the parameter $\Delta \phi_{\max }$. Predictions of NLO pQCD, corrected for NP effects, give a good description of the data except in the kinematic region of large dijet rapidity intervals $\left(y^{*}=\left|y_{\text {jet } 1}-y_{\text {jet } 2}\right| / 2>1\right)$ and small decorrelations $\left(\Delta \phi_{\max }=7 \pi / 8\right)$.

The ATLAS and CMS collaborations have also performed new measurements of the inclusive three-jet to two-jet cross section ratio at $\sqrt{s}=7 \mathrm{TeV}$. In the ATLAS measurement [12], the cross section ratio is measured as a function of both the leading jet $p_{T}\left(R_{3 / 2}\right)$ and the $p_{T}$ of each jet in an event $\left(N_{3 / 2}\right)$. The data agree well with NLO QCD predictions corrected for NP effects, except in the region $p_{T}^{\text {lead }}<140 \mathrm{GeV}$, where the pQCD predictions are lower than the $R_{3 / 2}$ measurements. From $N_{3 / 2}$, $\alpha_{s}\left(M_{Z}\right)=0.111 \pm 0.006$ (exp.) ${ }_{-0.003}^{+0.016}$ (theory) is extracted 

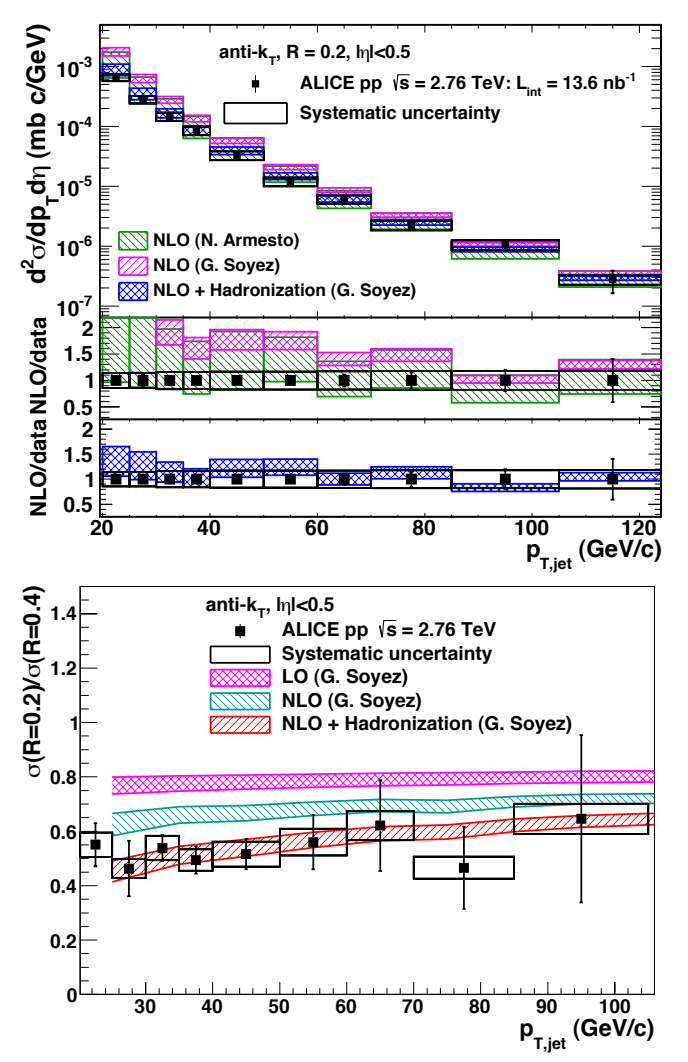

Figure 3. (Top) ALICE inclusive differential jet cross sections for $R=0.2$ together with NLO pQCD predictions, as described in Ref. [5] and references therein. (Bottom) Ratio of inclusive differential jet cross sections for $R=0.2$ and $R=0.4$.
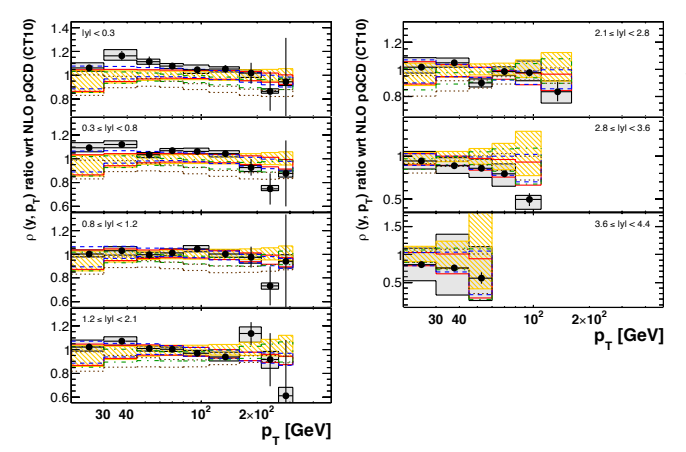

ATLAS

$\int L \mathrm{dt}=0.20 \mathrm{pb}^{-1}$

$\rho=\sigma_{\text {jet }}^{276 \text { TeV }} / \sigma_{\text {let }}^{\text {TeV }}$

anti- $k_{t} R=0.4$

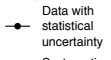

$\square$ Systematic NLO pQCD $\otimes$
non-pert. corrections

$\square$ ст10

二 mstw $2008^{2}$

$\because:$ NNPDF 2.1

::.: HERAPDF 1.5

….... ABM 11 NLO

Figure 4. ATLAS ratio of the inclusive jet cross section at $\sqrt{s}=$ $2.76 \mathrm{TeV}$ to the cross section at $\sqrt{s}=7 \mathrm{TeV}$, shown as a double ratio to the theoretical prediction calculated with the CT10 PDF set, as described in Ref. [6]. The double ratio is plotted as a function of jet $p_{T}$ in bins of jet rapidity for anti- $k_{T}$ jets with $R=0.4$. Many additional comparison plots are available in [6].

(see Fig. 9). The CMS analysis [13] measures the ratio $R_{32}$ as a function of the average transverse momentum of the two leading jets in the event, $\left\langle p_{\mathrm{T} 1,2}\right\rangle$. NLO QCD predictions for various PDF sets are in agreement with the data except for calculations using ABM11, which underestimate $R_{32}$ for $\left\langle p_{\mathrm{T} 1,2}\right\rangle<600 \mathrm{GeV}$. Measurements of $R_{32}$ have been used to determine $\alpha_{s}\left(M_{Z}\right)=0.1148 \pm 0.0014$ (exp.) \pm 0.0018 (PDF) ${ }_{-0.0000}^{+0.0050}$ (scale) (see Fig. 10). This
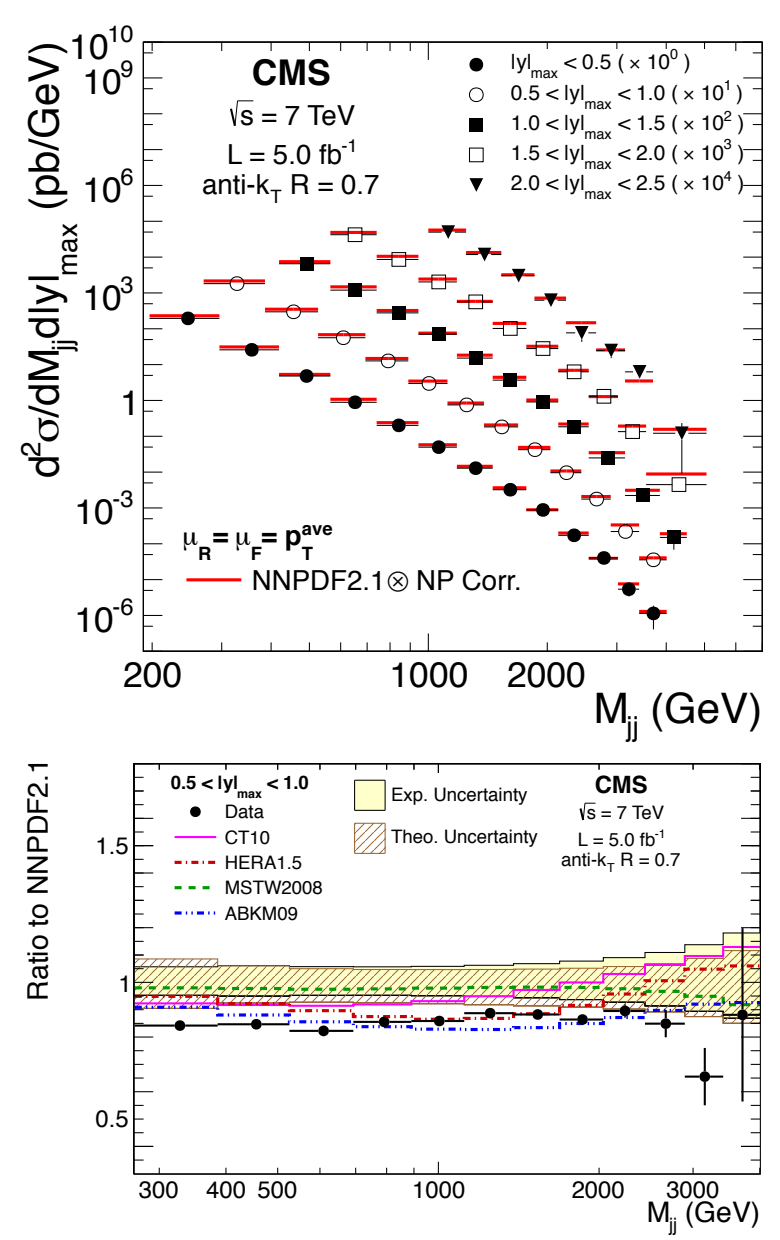

Figure 5. (Top) CMS dijet cross sections for five different rapidity bins for data (markers) and theory (thick lines) using the NNPDF2.1 PDF set, as described in Ref. [7]. (Bottom) Ratio of the dijet cross section to the theoretical prediction using the central value of the NNPDF2.1 PDF set for $0.5<|y|_{\max }<1.0$. Plots for four other rapidity ranges out to $|y|_{\max }=2.5$ are available in [7].

represents the first determination of the strong coupling constant from measurements at scales $Q$ of the order of $1 \mathrm{TeV}$.

\section{Jet Mass and Substructure}

The CDF collaboration has performed a study of the substructure of jets with $p_{T}>400 \mathrm{GeV} / \mathrm{c}$ at $\sqrt{s}=1.96 \mathrm{TeV}$ [14]. Distributions of the jet mass, angularity, and planar flow are measured with both the midpoint and anti- $k_{T}$ algorithms. The jet mass distribution above $100 \mathrm{GeV} / \mathrm{c}^{2}$ using both algorithms is in good agreement with PYTHIA as well as a simple NLO QCD jet function prediction (see Fig. 11). The results show that using jet mass is effective for separating jets produced through QCD and through $t \bar{t}$ production. In addition, studies of the angularity variable indicate that high-mass jets coming from light quark and gluon production are consistent with two-body final states, and it may be possible to use the planar flow variable to further reject high-mass QCD jets. 

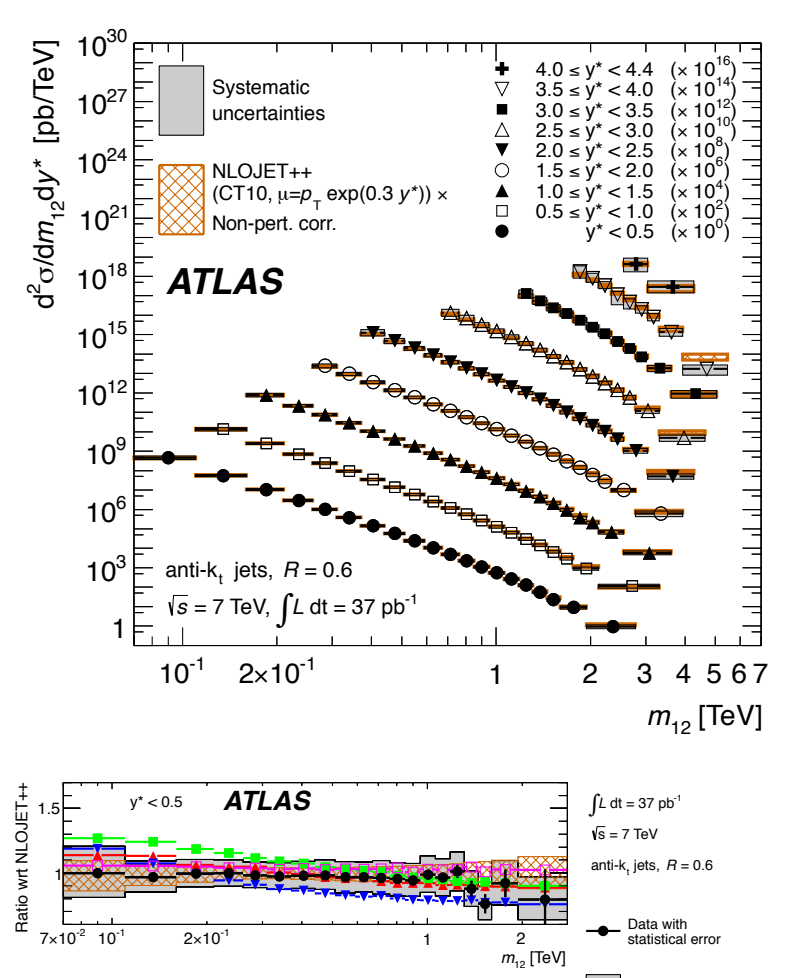

$\sqrt{s}=7 \mathrm{TeV}$

anti-k, jets, $R=0.6$
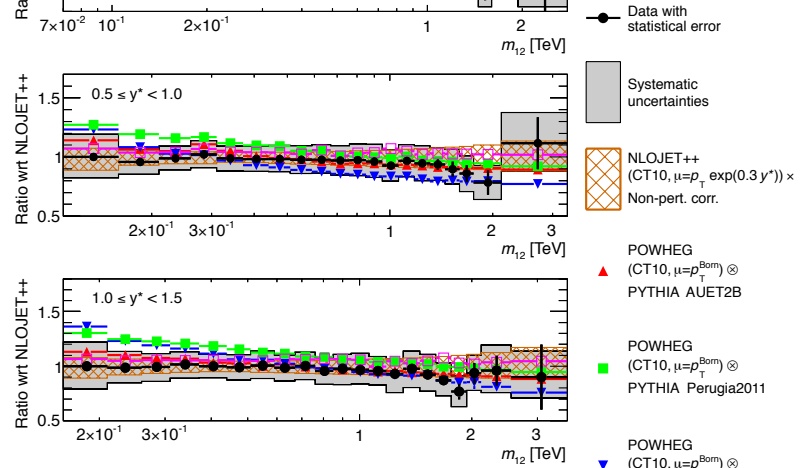

$\left(\mathrm{CT} 10, \mu=p_{T}^{\text {Borm }}\right) \otimes$
PYTHIA AUET2B

POWHEG

$\left(\mathrm{CT} 10, \mu=p_{\mathrm{T}}^{\mathrm{Bam}}\right) \otimes$

PYTHIA Perugia2011

POWHEG

HERWIG AUET2

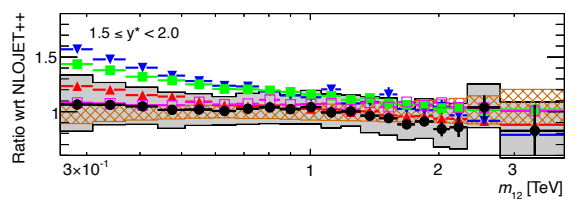

POWHEG fixed-order

POWHEG fixed-order
(CT10, $\left.u=p^{\text {Bom }}\right) \times$

Non-pert. corr.

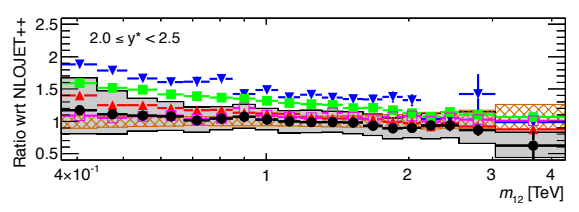

Figure 6. (Top) ATLAS dijet double-differential cross section as a function of dijet mass, binned in half the rapidity separation between the two leading jets, $y^{*}=\left|y_{1}-y_{2}\right| / 2$. The results are shown for jets identified using the anti- $k_{T}$ algorithm with $R=$ 0.6, as described in Ref. [3]. (Bottom) Ratios of the dijet doubledifferential cross section to the theoretical prediction obtained using NLOJET++ with the CT10 PDF set. The data are shown with ratios of POWHEG predictions showered using either PYTHIA or HERWIG to the NLOJET++ predictions, corrected for NP effects.
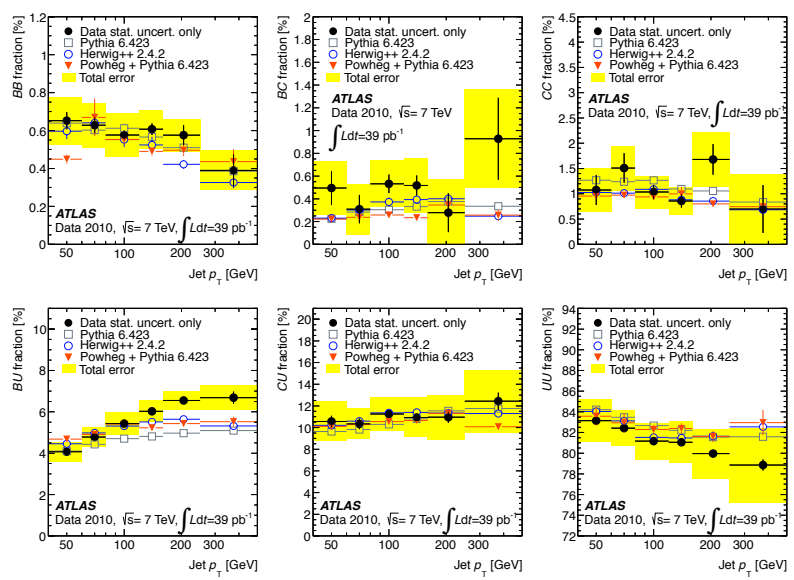

Figure 7. ATLAS unfolded dijet flavour fractions for each leading jet $p_{T}$ bin (black points) overlaid with predictions from Pythia, Herwig ++, and Powheg + Pythia, as described in Ref. [8].
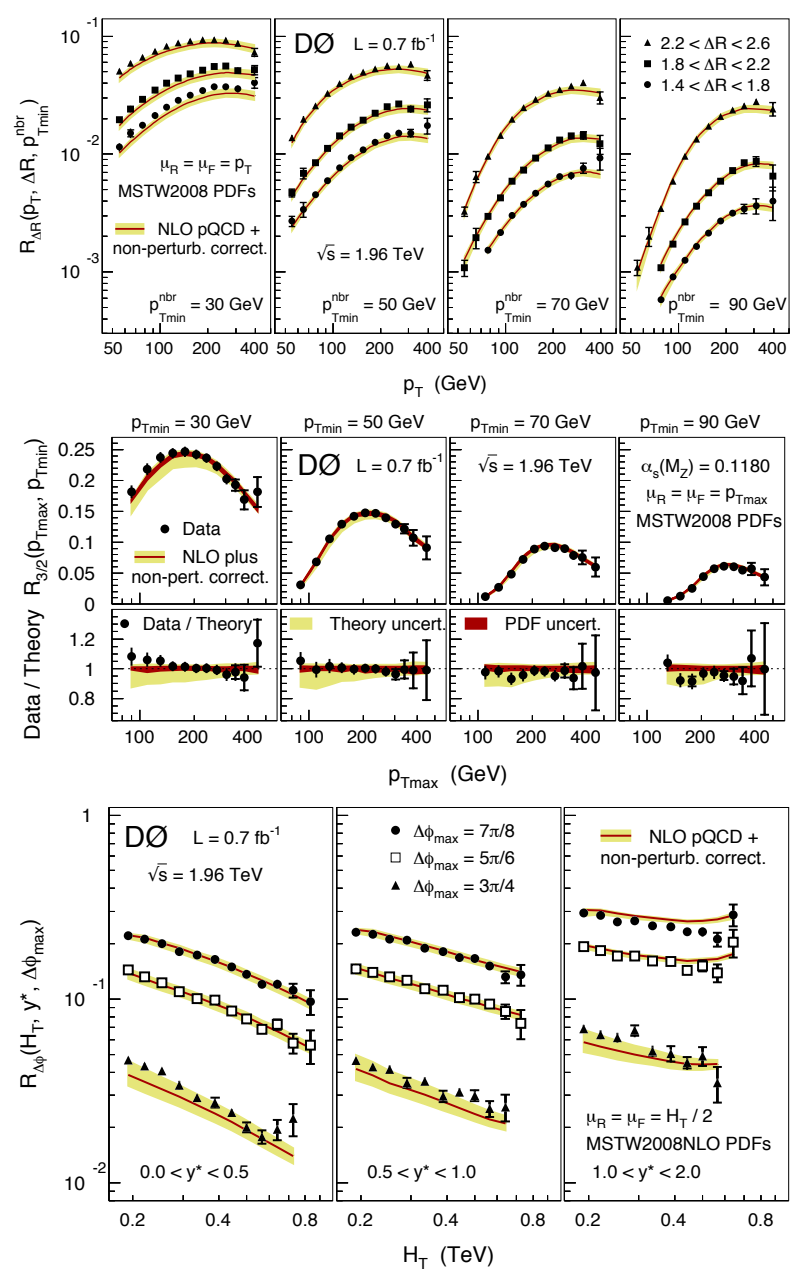

Figure 8. (Top) D0 measurement of $R_{\Delta R}$ as a function of inclusive jet $p_{T}$ for three different intervals in $\Delta R$ and for four different requirements of $p_{T \min }^{\mathrm{nbr}}$, as described in Ref. [9]. (Center) Measurement of $R_{3 / 2}$ as a function of the highest jet $p_{T}\left(p_{T \max }\right)$ for different $p_{T \text { min }}$ requirements, as described in Ref. [10]. (Bottom) $R_{\Delta \phi}$ as a function of $H_{T}$ in three different regions of $y^{*}$ and for three different $\Delta \phi_{\max }$ requirements, as described in Ref. [11]. 

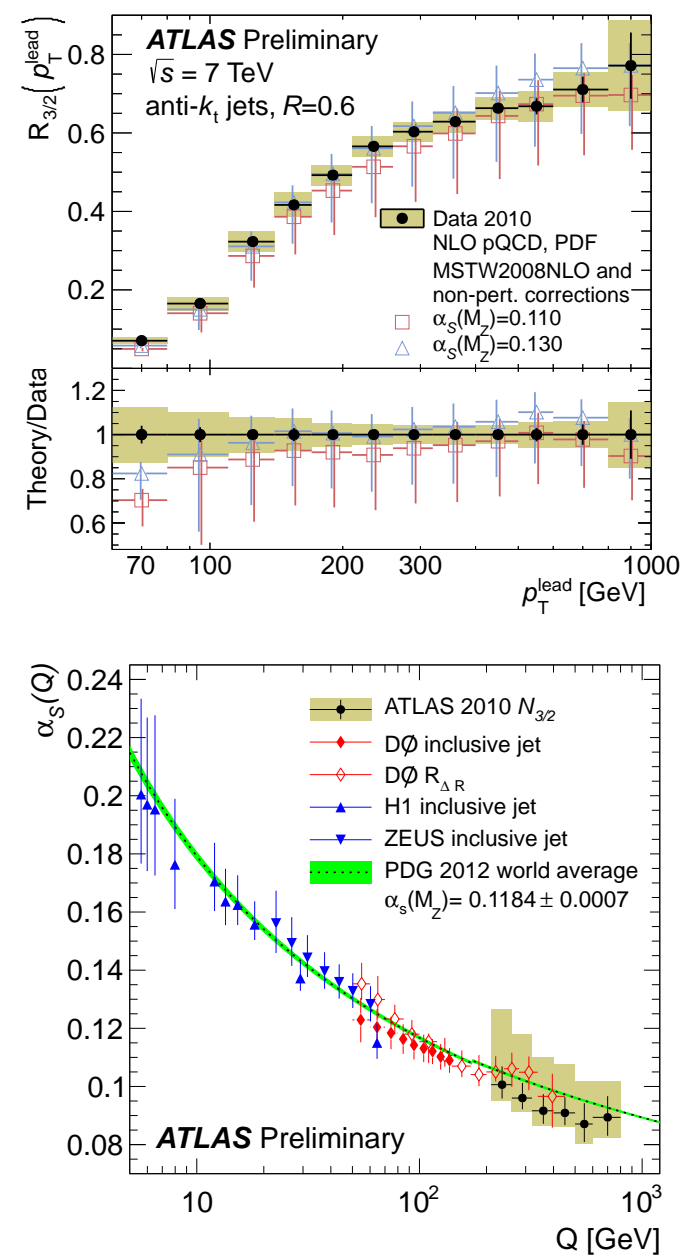

Figure 9. (Top) ATLAS measurement of $R_{3 / 2}$ at particle-level as a function of the leading jet $p_{T}$. (Bottom) Strong coupling constant $\alpha_{s}$ as a function of the renormalization scale $Q$, as described in [12] and references therein. The black points are extracted from the ATLAS $N_{3 / 2}$ measurement.

An ATLAS study of jet mass and the substructure of inclusive jets at $\sqrt{s}=7 \mathrm{TeV}$ also highlights the potential of using jet substructure techniques to identify the hadronic decays of boosted heavy particles [16]. Jet invariant mass, $k_{T}$ splitting scales, and $\mathrm{N}$-subjettiness variables are presented for anti- $k_{T} R=1.0$ jets and Cambridge-Aachen $R=$ 1.2 jets. Leading-order parton shower MC predictions for these variables are found to agree broadly with data (for example, see Fig. 12).

The CMS collaboration has investigated different jet "grooming" techniques using data collected at $\sqrt{s}=7 \mathrm{TeV}$ to distinguish merged jets of large $p_{T}$ from softer QCD gluon radiation [17]. Agreement with parton showers modeled with HERWIG ++ appears to be best for $p_{T}^{\mathrm{AVG}}>$ $300 \mathrm{GeV}$ and $m_{J}^{\mathrm{AVG}}>20 \mathrm{GeV}$ (see Fig. 14). More aggressive grooming procedures lead to somewhat better agreement between data and MC simulation.

In a new ATLAS analysis of jet shapes in top pair events at $\sqrt{s}=7 \mathrm{TeV}$ [15], the differential and integrated shapes of jets initiated by $b$ quarks from top quark decays are compared to jets from light-quark decays (see Fig. 13).
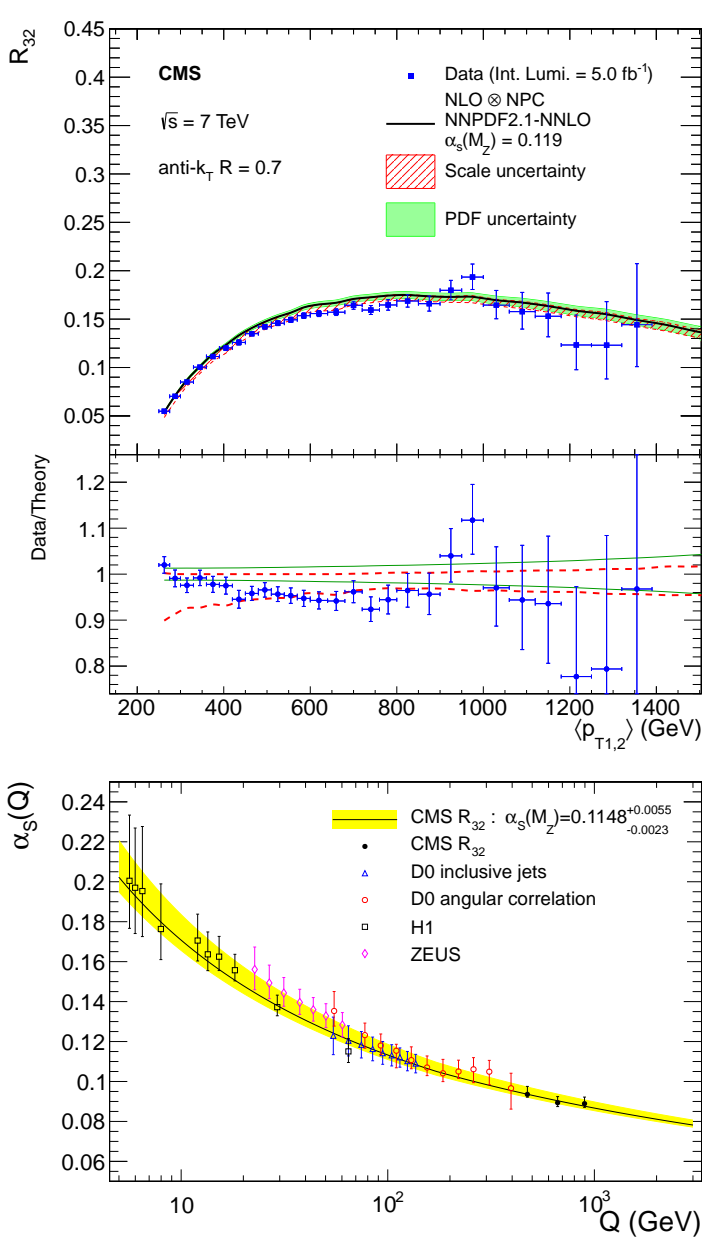

Figure 10. (Top) CMS measurement of $R_{32}$ with NLO predictions using the NNPDF2.1 NNLO PDF set, as described in Ref. [13]. (Bottom) The strong coupling $\alpha_{s}(Q)$ (solid line) and its total uncertainty (band) evolved from the CMS determination $\alpha_{s}\left(M_{Z}\right)=0.1148_{-0.0023}^{+0.0055}$ using a 3-loop solution to the renormalization group equation as a function of the momentum transfer $Q=\left\langle p_{\mathrm{T} 1,2}\right\rangle$, as described in [13] and references therein.

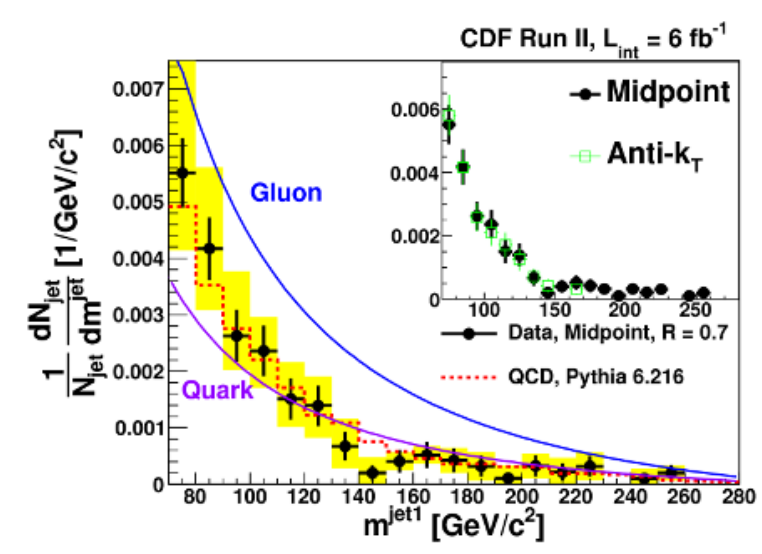

Figure 11. CDF normalized jet mass distribution for midpoint jets with $p_{T}>400 \mathrm{GeV} / \mathrm{c}$ and $0.1<|\eta|<0.7$, as described in Ref. [14]. The red dashed line shows the PYTHIA MC prediction, and the inset compares midpoint jets (full black circles) to anti- $k_{T}$ jets (open green squares). 

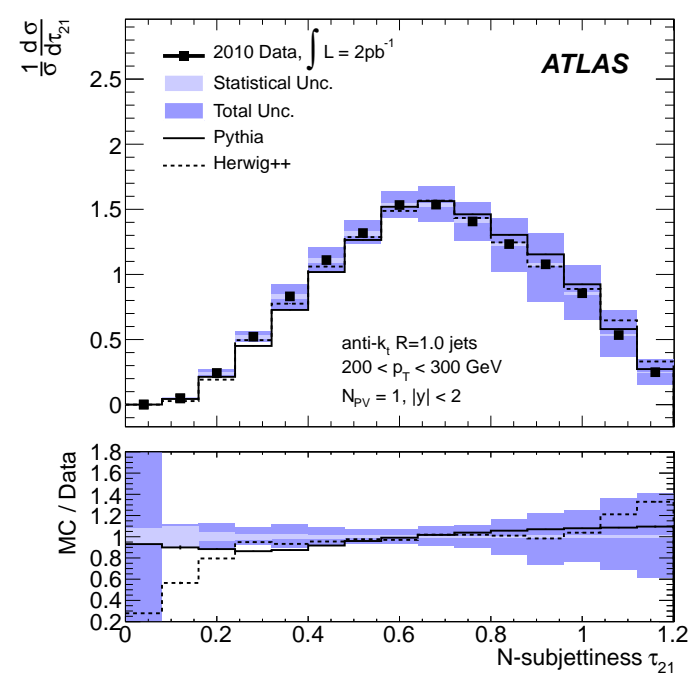

Figure 12. ATLAS normalized cross sections as functions of Nsubjettiness $\tau_{21}$ of anti- $k_{T}$ jets with $R=1.0$ in the $200-300 \mathrm{GeV}$ $p_{T}$ bin, as described in Ref. [16].

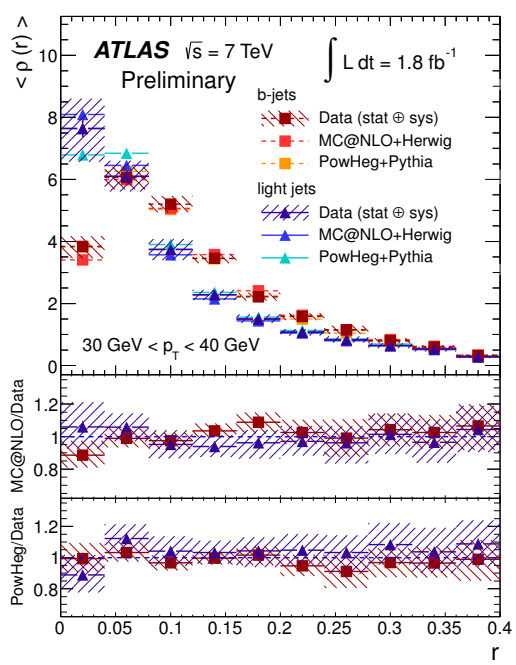

Figure 13. ATLAS differential jet shapes $\langle\rho(r)\rangle$ as a function of the radius $r$ at the particle level, compared to the MC@NLO + Herwig and Powheg + Pythia event generators for $30 \mathrm{GeV}<p_{T}$ $<40 \mathrm{GeV}$, as described in Ref. [15].

Light-quark jets are found to have a narrower distribution of momentum flow inside the jet cone than $b$-quark jets. Observations are in agreement with NLO calculations supplemented by parton showers.

\section{References}

[1] CDF Collaboration, Phys. Rev. D 78, 052006 (2008).

[2] D0 Collaboration, Phys. Rev. D 85, 052006 (2012).

[3] ATLAS Collaboration, Phys. Rev. D 86, 014022 (2012).

[4] CMS Collaboration, CMS Physics Analysis Summary CMS-PAS-SMP-12-012 (2013).
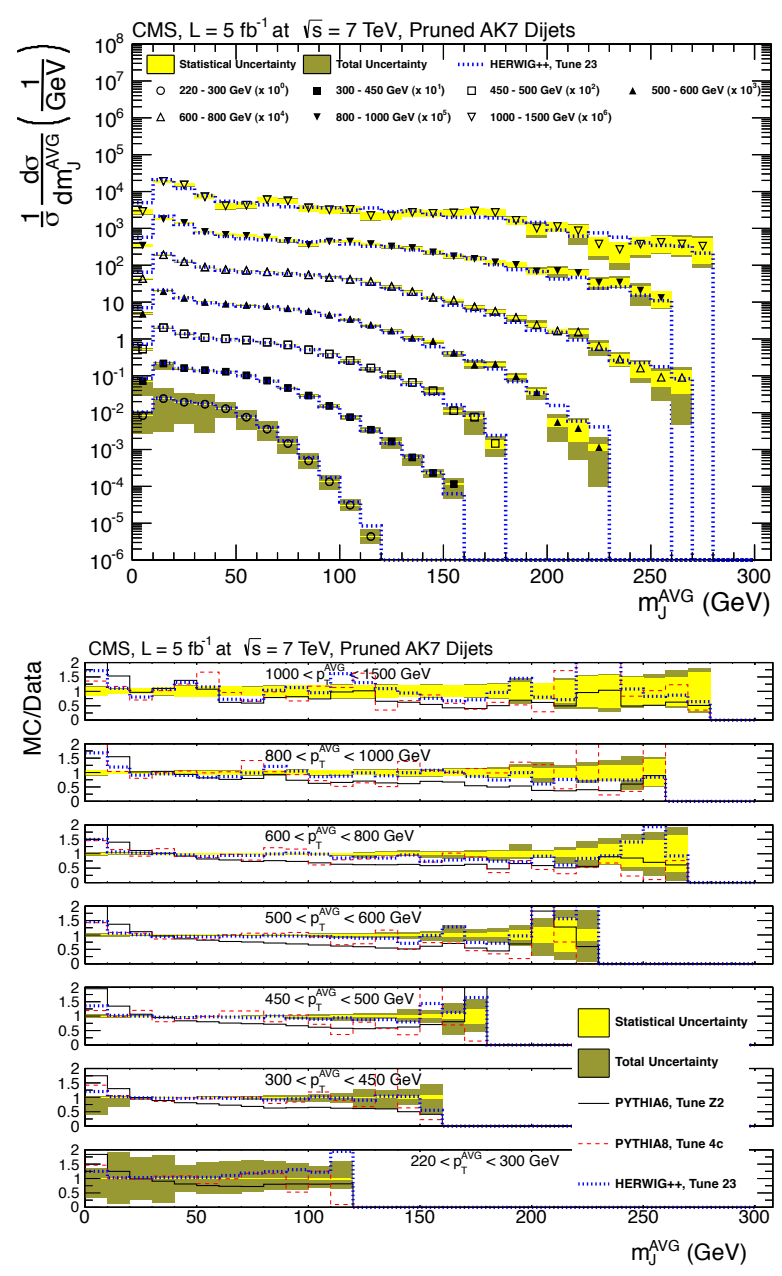

Figure 14. (Top) CMS unfolded distributions for the mean mass of the two leading jets in dijet events for reconstructed pruned $\mathrm{AK} 7$ jets, separated according to intervals in $p_{\mathrm{T}}^{\mathrm{AVG}}$ (the mean $p_{\mathrm{T}}$ of the two jets), as described in Ref. [17]. (Bottom) Ratio of MC simulation to unfolded distributions of the jet mass for pruned AK7 jets for seven bins in $p_{\mathrm{T}}^{\mathrm{AVG}}$, with comparisons to PYTHIA6, PYTHIA8, and HERWIG++.

[5] ALICE Collaboration, Phys. Lett. B 722, 262 (2013).

[6] ATLAS Collaboration, arXiv:1304.4739 (2013).

[7] CMS Collaboration, Phys. Rev. D 87, 112002 (2013).

[8] ATLAS Collaboration, Eur. Phys. J. C 73, 2301 (2013).

[9] D0 Collaboration, Phys. Lett. B 718, 56 (2012).

[10] D0 Collaboration, Phys. Lett. B 720, 6 (2013).

[11] D0 Collaboration, Phys. Lett. B 721, 212 (2013).

[12] ATLAS Collaboration, ATLAS Conference Note ATLAS-CONF-2013-041 (2013).

[13] CMS Collaboration, arXiv:1304.7498 (2013).

[14] CDF Collaboration, Phys. Rev. D 85, 091101 (2012).

[15] ATLAS Collaboration, STDM-2011-48 (2013).

[16] ATLAS Collaboration, J. High Energy Phys. 05128 (2012).

[17] CMS Collaboration, J. High Energy Phys. 05090 (2013). 